\section{Aortic dissection complicating carotid dissection and myocardial infarction}

\section{Chun-Cheng Chen ${ }^{1}$, Hung-Wen Tsai ${ }^{2}$ and Sung-Yuan $\mathrm{Hu}^{1,3-5 *}$}

\author{
${ }^{1}$ Department of Emergency Medicine, Taichung Veterans General Hospital, Taiwan \\ ${ }^{2}$ Division of Cardiovascular Surgery, Center of Cardiovascular Medicine, Taichung Veterans General \\ Hospital, Taiwan \\ ${ }^{3}$ School of Medicine, Chung Shan Medical University, Taiwan \\ ${ }^{4}$ Institute of Medicine, Chung Shan Medical University, Taiwan \\ ${ }^{5}$ Department of Nursing, College of Health, National Taichung University of Science and Technology, \\ Taichung, Taiwan
}

\section{Clinical image}

A 58-year-old hypertensive man presented to our institution with acute chest pain and dizziness. Electrocardiogram revealed inferior wall myocardial infarction with suspected right ventricular involvement (Figure 1A). Computed tomographic aortography (CTA) depicted ascending aortic dissection (AAD) with involvement of bilateral carotid, subclavian, and right common iliac arteries (Figure 1B). Replacements of aortic valve and ascending aorta with CABG (Ao-RSVG1-LAD and Ao-RSVG2-RCA) were conducted.

There are $5 \%$ - 8\% of ST-segment elevation and 15\% - 41\%

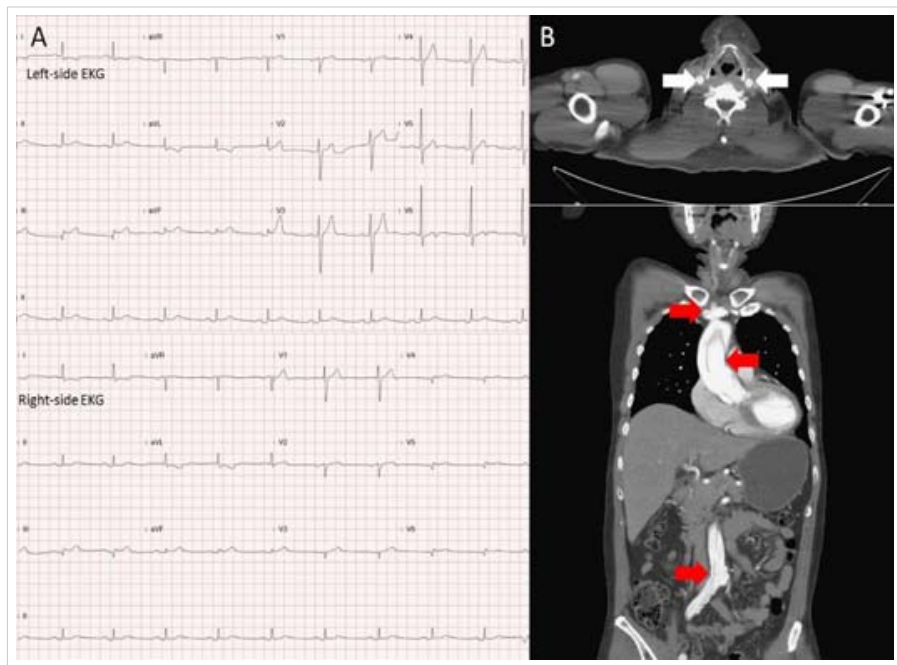

Figure 1: Left-side electrocardiogram (EKG) revealed ST-segment elevation in II, III, and aVF, with reciprocal changes in I and aVL, disclosing inferior wall myocardial infarction. Right-side (EKG) showed ST-segment elevation in V4, disclosing right ventricular involvement (Figure 1A). The axial and coronary views of computed tomographic angiography demonstrated dissection of bilateral carotid arteries (white arrows in Figure 1B) and dissection of ascending aorta with extending to subclavian and right common iliac arteries (red arrows in Figure 1B).

\section{Consent} the manuscript.

\section{References} 2014: 468295.

\author{
More Information \\ *Address for Correspondence: Sung-Yuan Hu, \\ Department of Emergency Medicine, Taichung \\ Veterans General Hospital, 1650 Taiwan \\ Boulevard Sect. 4, Taichung 40705, Taiwan, \\ Tel: +886 4 23592525; ext 3601; \\ Fax: +886 423594065 ; \\ Email: song9168@pie.com.tw \\ Submitted: March 15, 2021 \\ Approved: March 17, 2021 \\ Published: March 18, 2021
}

How to cite this article: Chen CC, Tsai HW, Hu SY. Aortic dissection complicating carotid dissection and myocardial infarction. $\mathrm{J}$ Clin Med Exp Images. 2021; 5: 003-003.

DOI: 10.29328/journal.jcmei.1001019

Copyright: @ 2021 Chen CC, et al. This is an open access article distributed under the Creative Commons Attribution License, which permits unrestricted use, distribution, and reproduction in any medium, provided the original work is properly cited.

(D) Check for updates

OPEn ACCESS

with dissection of the common carotid or subclavian artery in AAD [1-3]. CTA is a mandatory tool to confirm the diagnosis of AAD and its involvement [1]. Point-of-care ultrasound is an alternative tool to diagnose AAD if it is contraindicated or not available. AAD with involvement of coronary artery will lead to poor prognosis if delay intervention [1].

This study was approved by the Institutional Review Board of Taichung Veterans General Hospital (No. CE19152A).

This work was supported by grants from the Taichung Veterans General Hospital (Grant numbers: TCVGH-109 7202C). The funder had no role in the study design, data collection and analysis, decision to publish, or preparation of

1. Alsaad AA, Odunukan OW, Patton JN. Ascending aortic dissection presented as inferior myocardial infarction: a clinical and diagnostic mimicry. BMJ Case Rep. 2016; 20: bcr2016217543. PubMed: https://pubmed.ncbi.nlm.nih.gov/27999129/

2. Chen A, Ren X. Aortic Dissection Manifesting as ST-SegmentElevation Myocardial Infarction. Circulation. 2015; 131: e503-504. PubMed: https://pubmed.ncbi.nlm.nih.gov/26015467/

3. Deniz T, Dag E, Tulmac M, Azapoglu B, Alp C. What Lies behind the Ischemic Stroke: Aortic Dissection? Case Rep Emerg Med. 2014;

PubMed: https://pubmed.ncbi.nlm.nih.gov/25544904/ 